on his initial trip before he imparts that information, and is satisfied that they fully understand the matter; and it fre. quently is necessary to get an interpreter when one is dealing with Italians and other foreign-born people who do not speak the language.

We liave printed instructions. It would be interesting to find out how many of the hundreds given out are read intel. ligently and curefully. We are doing a great deal in the way of personal instruction of the family directly, or through an interpreter.

As to the plyysiciun's duties in case of contagious disenses, there is much to be said. There are all sorts of physicians in Chicago, as elsewhere. Some are antugonistic or careless; some are in sympatly with our work; but there is no one factor thut is of as great importance in this entire situation as the physician factor. It is up to the pliysiciuns to "get next" to the progressive work that is being done in this line; for if they don't, they will be left behind; progress will be made in spite of their indiflerence.

We not only put a policeman at the door, but frequently two: one at the front entrance and one at the rear; and two means four, because they work in relays, night and day. It is important; it brings the people to a realization of the fact that we mean business, and it has a salutary effect on them.

Common sense and tact are the greatest factors. You cannot compel the members of a Chicago community, or any large community, to observe strictly any precaution they lave never used before and perlaps have never heard of before. Many of these families have had a case of contagious discuse for the first time; they do not understand the situntion, and if you go at them abruptly you will lose out. This is where experience and tact come in. We try to have inspectors with com. mon sense and education, so fir as possible.

We allow nothing to come from the room without being thoroughly disinfected; and we supply bichlorid tablets; we make a solution in a wooden bucket right in the room, and not only are the linen bedding and the nurse's clothes disinfected, but also the bed-clothing, and the floor is mopped duily.

\section{THE TREATMENT OF WOUNDS}

\section{A FIRST ARTICLE *}

\section{ALEXIS CARREL, M.D.} NEW YOHK

In the actual condition of therapeutics, aseptic wounds generally heal in a few days. The more ambitious dreams of the surgeons of the pre-Listerian era have becn fulfilled. Nevertheless, we liave no right to believe that the treatment of wounds has reached its ultimate perfection. We must investigate whether or not it is possible to advance farther. In the treatment of wounds, we content ourselves by protecting the tissues against infection, and we leave to Nature the care of cicatrization. Wonld it not be feasible to act on the processes of reparation themselves and to activate them? The wounds which now heal in a few days could possibly be caused to heal in a few hours. The treatment of fractures would also be simplified. The development of methods for the stimulation of the growth of epithelial cells, for the inhibition or the activation of the proliferation of connective tissue, for the artificial production of osteogenesis, etc., would greatly improve the therapeutics of the ulcerations of the skin and of the lesions of peripheral nerves, bones and many other tissues or organs. 'This new evolution of surgery depends on the discovery, partial at least, of the laws of redintegration of tissues of mammals. Cicatrization and regeneration are the expression of the power to persist in its form with which all organisms are

* From the laboratories of the llockefeller Instltute for Medica Ilesearch. endowed. We are deeply ignorant of the nature of this function of redintegration. It is, as is the function of nutrition, a fundamental property of living matter. 'J'o know its nature is as impossible as to know the nature of life. Besides this knowledge would be useless. From a metaphysic standpoint it would be interesting to discover why a wound heals. But from a scientific standpoint, it is infinitely more important to know how it heals, because it would then be possible to find what stimuli start the complex mechanisms of the regeneration of the tissues. Therefore, the physiologic phenomena of cicatrization must be investigated. It is true that the power of redintegration escapes our methods of research. But the physico-chemical processes which this power, as a directing idea, coordinates and harmonizes in view of the morphologic reparation, can be brought into the field of experiment. We must, therefore, analyze the mechanisms which are instrumental in the cicatrization of a wound, the factors which modify their functions, the stimuli by which they are started, and the causes of their reciprocal cooperation to the common work. Perhaps it will become possible to use some of these agents for the artificial activation of the regeneration of tissues and the treatment of wounds.

\section{MECHANISMS OF THE REPALA'IION OF A CUTANLOUS WOUND}

Since many centurjes all surgeons know the anatomic processes of the cicatrization of a wound. On the open surface, granulations appear, and, by their contraction, bring closer to each other the edges of the epidermis. Then the epithelial cells wander on the granulous tissue and a new epidermis is formed. These phenomena can be divided into four periods: quiescent period, period of granulous retraction, period of epidermization and cicatricial period.

The experiments on which this article is based were performed cliefly on dogs. The cicatrization of wounds obtained by resection of a flap of skin was observel. The resected flap was of geometrical form, rectangular, trapezoidal or circular. In order that the edges of the old epidermis might be easily seen, I used black animals or I stained the edges of the wound with India ink. It was then possible always to distinguish the new from the old epidermis, and to follow accurately the varia. tions of the dimensions. The dressing consisted of talcum powder and gauze or warm paraffin. The wounds were kept as nearly aseptic as possible. When they became infected the results were discarded.

1. Quiescent Period.-The quiescent period extenıls from the time of the resection to the time of the beginning of the granulous retraction. During the first days the dimensions of the wound do not vary. If we represent graphically by a tracing the time of healing the successive distances between two points $A$ and $B$ talien on the opposite sides of a rectingular wound, the tracing during the quiescent period is horizontal. Surldenly it inclines downward. It is the beginning of the granulous retraction. Often the immobility of the edges of the wound during the quiescent period cease. rather suddenly; there is no period of transition and the active period of reparation starts immediately. The main characteristic of the quiescent period is the great variability of its duration. In some cases it lasts only one or two days, while in others it lasts four or five days.

2. Period of Granulous Retraction.-At the end of the quiescent period the edges of the wound begin to advance toward each other. The tracing of the con- 
sccutive distances between the points $A$ and $B$, taken on the opposite sides of a rectungular wound. shows a sudden inclination downwarl. Progressively, the inclination of the curve diminishes and, after a few days, it is almost horizontal. The reduction in size of the wound is very active during the first days of the period of granulous retraction. Then it becomes progressively slower until it comes to a standstill. This fact was observed long ago. It was believed that the activity of the gramulations depended on their age, while it depends really on the dimensions of the wound.

By measuring the rate of reparation of a rectangular wound, I found that it diminishes progressively from the beginning to the end of the period of granulous setraction. The rate when the wound is 60 or $70 \mathrm{~mm}$. is about 9 or $10 \mathrm{~mm}$. for twenty-four hours. When the wound is one of $40 \mathrm{~mm}$. the rate is about $3 \mathrm{~mm}$. When the dimensions of the wound are only $20 \mathrm{~mm}$. the rate becomes very slow. In all wounds the rate becomes about zero when the elges have reached a distance of about 10 or $15 \mathrm{~mm}$. from each other. It is therefore certain that these differences in the rate of reparation are functions of the size of the wound.

By observing on the same animals large and small wounds, I could see during the same period the larger wound diminishing with a much greater speed than the smaller wound. For instance, two rectangular wounds (Experiment 176 ) were made on the same animal. The transverse dimension of the one was $66 \mathrm{~mm}$. and of the other $26 \mathrm{~mm}$. During the first forty-eight hours of the period of gramulous retraction, the larger wound diminished $20 \mathrm{~mm}$. and the smaller $4 \mathrm{~mm}$. On trapezoidul wounds, it was observed also that the reduction in size of the smaller side is very much slower than the rerluction undergone by the larger side. On circular wounds, made on the same animals with cutting tubes 1 and $2 \mathrm{~cm}$. in diameter, the same phenomena were olsserved. Many other experiments lave been performed. It is certain that the rate of reparation of the granulous period is directly proportional to the size of the wound: that is, to the effort to be accomplished in order to hring alout the redintegration of the parts. It must be noticed that during the period of granulous retraction, the redintegration of the skin of mammals follows the law discovered by Spallanzani on the salamander. If the tail of a fish" or shlamanter is cut off near its base, the new part grows faster than when the tail is eut off nearer the tip. The new puit which arises from the basal cut grows more rapidly at first and more slowly later. The rate of regeneration is proportional to the importance of the work to be done. It is remarkable that, on mammals, the reparation of the skin, which is brought about by a rery different mechanism, obeys the same general law.

The period of granulous retraction plays a very important rôle in the healing of the midrlle-sized and the large wounds. 'Thus, a wound of 60 or $80 \mathrm{~mm}$. an be reduced to one-third and one-fourth of its primitive size. A wound of $30 \mathrm{~mm}$. can be reduced to one-half its size. The importance of the granulous period is less for the small wounds. 'The effort of the granulations on a wound of about $15 \mathrm{~mm}$. reduces its dimensions to three-quarters or even less of its original size.

The end of the granulous period corresponds to the beginning of the epithelial wandering from the edges of the wound. For a wound of albout 30 or $40 \mathrm{~mm}$. the retraction becomes exceedingly slow and even stops completely when the distance between the edges has been reduced to 10 or $15 \mathrm{~mm}$. When the wound is larger, the retraction stops often when its dimensions are still 20 or $25 \mathrm{~mm}$. In that case the epithelial wandering is very slow and often the result is an uleer.

It seems that the epithelial wandering on the surface of the granulations stops immediately their retraction. The epidermization not only coincirles with the end of the period of granulous retraction, but it causes it. In the corner of a granulating rectangular wound I deposited a small square graft of skin. After a lew days the graft was found surrounded by the normal skin of the edge and the wound had assumed angin a perfectly rectangular appearance. This shows that the retraction stopped at the level of the graft, while it still went on in the other parts of the wound. In another case, the epidermization of the upper part of a large square wound was stimulated by a graft, while the lower part remained without epithelimm. It was then observed that the distunce between the India-ink-stained edges of the old epidermis was still diminishing in the lower part, while it increased in the upper part. 'The shatpe of the square wound became trapezoidal. Sometime after complete epidermization it became square again. It is, therefore, certain that the epidermization inhibits the retractive function of the granulations. When the epidermization takes place early, the scar is large and thin. When the epidermization is late, the granulations undergo a stronger retraction and the scar is thick and comparatively smaller.

'The function of the granulous period is also to pre. pare the surface of the wound for the wandering of the epithelial cells. But it seems probable that its main rôle is to bring the edges of the wound to a certain distance-about 10 or $15 \mathrm{~mm}$. in the dog. It is shown by the fact that if the wound is only $10 \mathrm{~mm}$. wide, practically no retraction occurs. It does not oceur because it would be useless, since at the distance of $10 \mathrm{~mm}$. the next mechanism of the reparation, that is, the epithelial wandering, can take place easily, as will be shown later.

3. Period of Epidermization.-On a rectangular wound, the edges of which are stained with Indin ink, it is easy to detect the beginning of the perior of epidermization. 'The new epithelium spreads at first very slowly on the surface of the granulations. It is clifficult to locate exactly the free edge of the new epiclermis. Nevertheless, by using paraffin dressing, it is possible to see with certainty in a few cases the edge of the wandering epithelium. The new epidermis is cxcedingly delicate and a great many external factors interfere with its growth. The best inedium for it growth is certainly coagulated fibrin, which can be obtained by using as a dressing paraffin of a certain consistency and meltingpoint.

The time of the beginning of epidermization does not depend on the age of the wound but on its dimensions. If the wound is large, the epidermization is tarly. It occurs very much earlier when the wound is smaller.

By measuring the distance between two points taken on the free edge of the new epidermis of a rectangular wound, it was found that the growing of the epithelium is exceedingly slow if the distance is more than 12 or $15 \mathrm{~mm}$. But if the edges are located less than $10 \mathrm{~mm}$. from each other, the epithelium wanders more quickly on the granulations. When the free elges of the epithelium are closer, the rate of cicatrization is very much faster. I found, in one case, a rate of $2.5 \mathrm{~mm}$. for a distance of $5 \mathrm{~mm}$. 'The rurve representing the positions of the two points runs at first almost hori\%ontal and progressively inclines itself downward with an accolerated rate. 
When at the end of the granulous retruction of a large wound, the edges of the old epidermis are still at a distance of 20 or $25 \mathrm{~mm}$. the new cpidermis cannot spread on the granulations and the cicatrization of the wound comes to a standstill.

It seems that the time of the epidermization and its rate depends mainly on the dimensions of the wound. This point has been ascertained by several other sets of experiments.

By observing trapezoidal wounds it was found that the smaller side about $8 \mathrm{~mm}$. wille was completely epidermized while the larger side about $20 \mathrm{~mm}$. wide presented an epithelial bund of about $2 \mathrm{~mm}$. along the edges of the old epidermis. On irregular wounds the epidermization begins alwars on the points where the edges are closer to each other. In lozenge-shaped wounds the epidermization begins in the acute angles and the wound becomes an ellipse. On several kinds of trapezoidal wounds it was always found that the epidermization begins sooner and spreads more quickly between the points which are separated by the shortest distance.

'Therefore, it appears that the law of reparation by epiclermization is absolutely different from the law of separation by granulous retraction. The rate of the epidermization is inversely proportional to the dimensions of the wound. It is very slow when the distance between the edges of the wound is more than 10 or 15 mm. 'The maximal activity of the epidermization seems to take place when the cicatrization is nearly complete, and when the edges of the new epithelium are very close to each other.

4. Cicalricial Period.-The dimensions of the scar can easily be measured when the edges of the old epidermis are stained with India ink, or when the aninial is black. It was found that the scar of a large wound is comparatively smaller than that of a small wound. On the same animal, two wounds of $66 \mathrm{~mm}$. and of 26 $\mathrm{mm}$. were observed. The $66 \mathrm{~mm}$. wound gave a scar of $22 \mathrm{~mm}$. and the $26 \mathrm{~mm}$. wound a scar of $13 \mathrm{~mm}$. 'l'he scar of the large wound was only one-third the size of the wound, while the sear of the small wound was onelualf the size of the wound. If the wound is still smuller, 10 or $12 \mathrm{~mm}$., the scar is almost the same size as the wound. This is the natural result of the law of granulous retraction.

The evolution of the scar is very slow and the ricatricial period of a wound very long. As soon as the epidermization is conıpleted, the distance between the points $A$ and $B$ of the edges of the old epidermis grows greater. The tracing shows a slight movement upward of the line representing the different values of the distance $A$ and $B$. The points $A$ and $B$ have a tendency to go back to their former position. 'This progressive enlargement of the scar lasts for a long time and its result should be a complete redintegration.

The mechanisms which are instrumental in the cicatrization of a wound are coordinated in such a way that the reparation is continuous and progressive. Nevertheless, the reparation presents phases of maximum and minimum activity during which the rate is higher or lower. During the quiescent period, the end of the period of granulous retraction and the beginning of the period of epidemization, the rate of the reparation is slow. It is maximum at the begrinning of the period of granulous retraction and at the end of the period of epidermization. The two mechanisms are adapted to the healing of small and middle-sized wounds, the width of which is not over $40 \mathrm{~mm}$. In a wound 30 or $40 \mathrm{~mm}$. in width or smaller, the retraction of the granulations is very efficient, since it can quickly bring the edges to a distance of 10 or $15 \mathrm{~mm}$. This distance is very favorable to the epidermization. Therefore, at the same time when the rate of reparation by granulation becomes very slow, the epidermization starts and the reparation goes on without interruption, although by a different mechanism. But if the wound is larger. 60 or $70 \mathrm{~mm}$., the retraction of the granulations cannot bring the edges to the minimum distance. They remain at a distance of about $20 \mathrm{~mm}$. and the repriation comes to a standstill because the epidermization cannot take place easily under these conditions. 'The mechanisms are very officient for the healing of the injuries to which the animals are exposed in their every-clay life. But they do not work as ratisfactorily for the larger wounds.

\section{VAPOR ANESTHESIA APPARATUS}

\section{JAMES T., GWATHMEY, M.D.} New YokK

In 1905 I first presented my apparatus for vanor anesthesia to the medical profession. At that time it seemed quite complicated. It consisted of three lottles connected by tubing, one of which was for ether, one for chloroform and the other being a bottle containing hot water or hot neutral oil for warming the vapor, and having a tube through which the vapor was delivered to the patient. Each bottle was provided with a stop-cock, and a mixture of chloroform and ether could he given. The ether bottle contained a drum for assisting in vaporizing the ether. I have perfected the different parts and

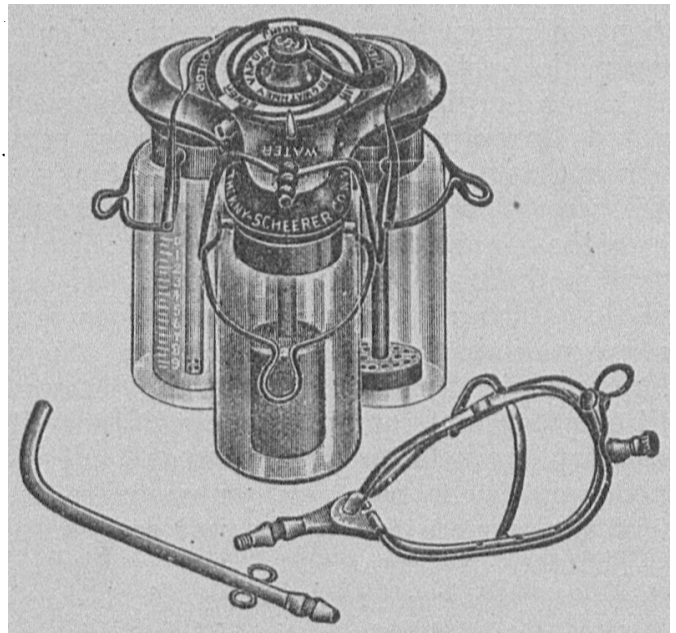

Iig. 1.-Thls shows the three bottles of the vaporizing apparatus, with the single stopeck. The figure at the right below shows the tubular frame of the mask through which the vapor passes to the rawze covering. The tube at the ieft below is used in dellvering the inesthetle in operations about the mouth or throut in which the mask canot be used.

now present a simplified apparatus consisting of three bottles with one stop-cock. 'The drum in the ether bottle has been modified (as suggested by Dr. Charles F. Boys, Kalamazoo, Mich.), so that it now vaporizes all of the ether, and it does not necessitate refilling the bottle as often as before. A small bottle holding 10 drams of chloroform has also been placed within the chloroform bottle. This is enough chloroform for a long operation. 'The end of the tube in the chloroform hottle has been closed and a number of ninhole perforations made at the bottom of this tube, so that it is impossible to waste 1l:e chloroform as the air passes through. The last improre- 